\title{
Actividad antifúngica del aceite esencial y extracto alcohólico de mentha piperita contra malassezia furfur
}

Antifungal activity of Mentha piperita's essential oil and alcoholic extracts againts Malassezia furfur:

\author{
Bravo Torres Idania \\ Erazo Yagual Rosa \\ García Larreta Soraya
}

Fecha de recepción: 13 de mayo del 2021

Fecha de aceptación: 27 de junio del 2021 


\title{
Actividad antifúngica del aceite esencial y extracto alcohólico de mentha piperita contra malassezia furfur
}

\author{
Antifungal activity of mentha piperita's essential oil and alcoholic extracts \\ againts malassezia furfur \\ Bravo Torres Idania ${ }^{1}$. Erazo Yagual Rosa ${ }^{2}$. García Larreta Soraya ${ }^{3}$.
}

Como citar: Bravo, I., Erazo, R., García, S., (2021). Actividad antifúngica del aceite esencial y extracto alcohólico de mentha piperita contra malassezia furfur, Revistc Universidad de Guayaquil. 132(2), 17-26. DOI: https://doi.org/10.53591/rug.v133i2.1380

\begin{abstract}
RESUMEN
Debido a la gran incidencia de enfermedades de la piel como la dermatitis seborreica y las reacciones adversas asociadas a medicamentos que se usan como tratamiento para estos trastornos, el objetivo de este estudio es evaluar la actividad antifúngica de los extractos alcohólicos y aceite esencial de las hojas de Mentha piperita frente al hongo Malassezia furfur. Los procedimientos de extracción consistieron en procesos de maceración, secado a bajas temperaturas y filtración del extracto alcohólico al 70\% obtenido de las hojas, así como el método por arrastre de vapor en donde una vez condensada la esencia, esta se recolecto y separó de la fracción acuosa para la obtención del aceite esencial. Los halos de inhibición del microorganismo se determinaron por metodología de difusión de discos Kirby Bauer, utilizando una cepa pura de Malassezia furfur ATCC que fue sembrada en una placa de suspensión homogénea del liofilizado del microorganismo, en Agar Sabouraud Dextrosa enriquecido con 10\% de Aceite de Oliva Extra Virgen por un periodo de 7 días, en incubación a 30 ${ }^{\circ} \mathrm{C}$. Para la determinación de la actividad antifúngica se realizaron diluciones a diferentes concentraciones tanto para el aceite esencial como para el extracto alcohólico, y los resultados fueron establecidos por grados de sensibilidad. Los resultados indicaron que los aceites esenciales de la Mentha piperita presentaron halos de inhibición mayores que los del extracto alcohólico frente al microorganismo M. furfur principal causante de enfermedades como la caspa seborreica.
\end{abstract}

Palabras clave: Mentha, dermatitis, aceite esencial, Malassezia.

1 Estudiante de la Facultad de Ciencias Químicas, Universidad de Guayaquil, Ecuador. Correo electrónico: idiana.bravot@ug.edu.ec. 2 Estudiante de la Facultad de Ciencias Químicas, Universidad de Guayaquil, Ecuador. Correo electrónico: rosa.erazoy@ug.edu.ec

3Magister en Diseño Curricular, Docente de la Facultad de Ciencias Químicas, Universidad de Guayaquil, Ecuador. Correo electrónico: soraya.garcial@ug.edu.ec 


\begin{abstract}
Due to the high incidence of skin diseases such as seborrheic dermatitis and the adverse reactions associated with medications used as treatment for these disorders, the objective of this study is to evaluate the antifungal activity of alcoholic extracts and essential oil from the leaves of Mentha piperita against the fungus Malassezia furfur. The extraction procedures consisted of maceration processes, drying at low temperatures and filtration of the $70 \%$ alcoholic extract obtained from the leaves, as well as the steam drag method where once the essence was condensed, it was collected and separated from the aqueous fraction to obtain essential oil. The aura inhibitors of the microorganism were determined by Kirby Bauer disc diffusion methodology, using a pure strain of Malassezia furfur ATCC that was seeded in a homogeneous suspension plate of the microorganism's lyophilisate, in Sabouraud Dextrose Agar fortified with $10 \%$ extra virgin olive over a 7 days period, in incubation at $30^{\circ} \mathrm{C}$. The determination of the antifungal activity, dilutions was made at different concentrations both for essential oil and the alcoholic extract, the results were established by degrees of sensitivity. These results indicated that the essential oils of Mentha piperita showed bigger aura inhibitors against the microorganism M. furfur, the main cause of diseases such as seborrheic dermatitis, in comparison with the alcoholic extracts.
\end{abstract}

Keywords: Mentha, dermatitis, essential oil, Malassezia.

\title{
INTRODUCCIÓN
}

El presente trabajo de investigación abarca el estudio de la actividad antifúngica que presentan los extractos alcohólicos y el aceite esencial de la Mentha piperita frente al desarrollo de la levadura Malassezia furfur principal causante de la caspa seborreica y que además está asociada con otras formas de dermatitis. En las últimas décadas se ha observado un incremento del uso de aceites esenciales provenientes de plantas medicinales, para fines farmacéuticos.

La actividad antifúngica ha sido determinada en base a la capacidad inhibitoria que posee el aceite esencial y extracto alcohólico de las hojas de M.piperita frente al crecimiento de la levadura Malassezia furfur. En esta investigación se estudian qué concentraciones de los halos de inhibición resultan aptos para evitar el crecimiento de este microorganismo, y cuáles metabolitos secundarios son los principales responsables de este efecto.

Los métodos de análisis que hemos utilizado para determinar la actividad antifúngica de $M$. piperita frente a $M$. furfur consisten en el método de difusión de discos de Kirby-Bauer, el cual 
implicácu un análisis de susceptibilidad para determinar la resistencia de agentes patógenos aeróbicos y bacterias anaerobias facultativas frente a varios compuestos antimicrobianos. Otros métodos utilizados durante esta investigación se basan en procesos de desecación, filtración y maceración para la obtención de los extractos hidroalcohólicos, y destilación por arrastre de vapor para la obtención del aceite esencial.

\section{METODOLOGIA}

Esta investigación es de tipo experimental, in vitro y exploratoria con enfoque cuantitativo, la cual se fundamentó en la obtención de evidencias reales mediante el uso de las técnicas ya establecidas con el propósito de determinar la actividad antifúngica del extracto alcohólico y aceite esencial de las hojas de Mentha piperita. La muestra empleada en el presente estudio de investigación son las hojas de Mentha piperita las cuales fueron obtenidas en el Mercado Casuarina ubicado en la Av. Casuarina, norte de Guayaquil. Esta planta fue elegida por su actividad positiva frente a Malassezia furfur según revisiones bibliográficas.

Los criterios de inclusión fueron cepas puras de Malassezia furfur sin contaminación, hojas de menta frescas, verdes, lisas sin daño alguno, extracto alcohólico y aceite esencial de Mentha piperita en las concentraciones de 12500ppm, 10500ppm, 8500ppm, 6500ppm, 4500ppm y 2500 ppm.

\section{Procedimiento para la obtención del extracto alcohólico al $70 \%$ de Mentha piperita (menta)}

Se seleccionaron las mejores hojas de la planta de la menta y se desecharon las hojas secas, quemadas, rotas, se lavaron con agua para la eliminación de impurezas y se dejaron reposar con agua destilada durante diez minutos. Las muestras húmedas fueron sometidas a un proceso de secado a bajas temperaturas por largo períodos de tiempo.

Se realizaron los extractos hidroalcohólicos al 70\% (Etanol G.R. J.T. Baker) por proceso de maceración del material seco obtenido $(10 \mathrm{~g})$ luego del secado de $60^{\circ} \mathrm{C}$ por 36 horas, durante 48 horas y luego se filtró por papel filtro Whatman para eliminar material extraño. El extracto obtenido se llevó al rotavapor (Buchi) en el cual se eliminó el solvente del extracto hasta su sequedad. Luego se llevó a volumen con Etanol G.R. y a partir de este extracto se realizaron las diluciones.

\section{Procedimiento para la obtención del aceite esencial de Mentha piperita (menta)}

La obtención del aceite esencial se realizó por destilación por arrastre de vapor en el que la hojas frescas y cortadas en trozos pequeños se encerraron en una trampa tipo Clevenger y se sometieron a una corriente de vapor de agua sobrecalentado, luego de ser arrastrada se condensa la 
essenciala, se recolecta y se separa de la fracción acuosa, este proceso tuvo una duración de tres horas.

\section{Activación de Malassezia Furfur ATCC 14521 y difusión de discos}

Se sembró por el método de dispersión en placa la suspensión homogénea del liofilizado de Malassezia Furfur en Agar Sabouraud Dextrosa con 10\% de Aceite Oliva Extra Virgen, durante 7 días a $30^{\circ} \mathrm{C}$ (incubación).

A partir de un cultivo de Malassezia furfur durante 7 días a $30^{\circ} \mathrm{C}$, se seleccionan 4 a 5 colonias y se prepara una suspensión en solución estéril al 0,9\% hasta alcanzar una concentración equivalente a 0,5 McFarland (1-5x106UFC/mL). Finalmente se realizó una difusión de los discos tanto en aceite esencial como en extracto alcohólico de M.piperita los cuales pasaron por un proceso de incubación que tardó 7 días, para posteriormente analizar los resultados con respecto a los halos de inhibición obtenidos. Se trabajó por cudrip

\section{DESARROLLO}

En el 2018 Lee Min Young y sus compañeros llevaron a cabo un estudio in vitro y ensayo clínico con la finalidad de evaluar el efecto inhibidor de diferentes extractos alcohólicos, aceites esenciales naturales y extracto de fruta sobre las especies de Malassezia furfur y Malassezia sympodialis midiendo la concentración mínima inhibitoria (MIC), la concentración mínima fungicida (MFC) y utilizando el método de difusión por disco, usando los extractos y aceite esencial de plantacomo la menta, lavanda y romero, los resultados definieron que la actividad antifúngica de los agentes de lavanda y cidra fue relativamente menor que los de la menta en MIC Y MFC (1).

Los aceites esenciales están compuestos principalmente de monoterpenos y sus derivados en proporciones del 76-90\% pero varían según la especie. En el caso de las proporciones de mentol y mentona del aceite esencial, se encuentran en una proporción del 50\% en Mentha piperita, mientras que el terpenoide carvona se encuentra en $70 \%$ de aceite esencial de hierbabuena o Mentha spicata (2).

La sociedad coreana de micología médica ha definido a Malassezia furfur como una levadura dismórfica lipofílica que forma parte de la mucosa normal de la piel humana y que además causante principal de la caspa y dermatitis seborreica. También está asociada con otras enfermedades como la pitiriasis versicolor, foliculitis por Malassezia, dermatitis atópica y psoriasis (3).

La eficacia antifúngica de Mentha piperita fue corroborada por Tullio Vivian et al. en el año 
2019 eñ su estudio "Evaluación de la actividad antifúngica del aceite esencial de Mentha piperita (Lamiaceae) y su interacción sinérgica con los azoles" en donde se demostró que el aceite esencial ejerce actividad fungicida contra levaduras del género Malassezia, estableciendo que puede servir para el tratamiento en afecciones como la caspa (4).

En 2017 Lee Jeong-Hyun comprobó la actividad anti Malassezia furfur y Malassezia pachydermatis del limoneno (uno de los componentes del aceite esencial de menta) revelando resultados positivos indicando que podría ser potencialmente eficaz para controlar infecciones causadas por Malassezia furfur (5).

Los diferentes estudios y referencias mencionadas anteriormente, fueron comparados con los que se obtuvieron durante esta investigación, obteniendo un resultado positivo en cuanto a la efectividad del aceite esencial y extracto alcohólico frente al crecimiento de Malassezia furfur. El aceite de Mentha piperita presentó un comportamiento directamente proporcional al igual que los estudios pasados, siendo que a las concentraciones de 2500 ppm, 4500 ppm, 6500 ppm y 8500 ppm fueron sensibles frente al microorganismo.

Con respecto a los extractos alcohólicos de Mentha piperita a la concentración de 5000 ppm este presentó actividad antifúngica nula de acuerdo a Duraffourd. Mientras que, para las concentraciones a partir de 10000, 20000, 30000, 40000 y 50000 ppm presentaron sensibilidad límite. 


\section{RESULTADOS}

En la tabla 1se muestran las medias de los halos de inhibición (expresadas en mm) de las diferentes concentraciones (expresadas en ppm) utilizadas en el ensayo del aceite esencial de Mentha piperita con sus respectivas desviaciones estándar. El comportamiento del halo de inhibición del aceite esencial de menta en la concentración de $2500 \mathrm{ppm}$ fue de $9.25 \mathrm{~mm} \pm 0.50$. Se apreció un crecimiento directamente proporcional con las concentraciones de 4500 ppm, 6500 ppm y 8500 ppm obteniendo medias de $9,50 \mathrm{~mm} \pm 0.57,10,75 \mathrm{~mm} \pm 0.50$ y $11,50 \mathrm{~mm} \pm 0.50$ respectivamente. Además, para las concentraciones de 10500 ppm y 12500 ppm se obtuvieron medias de 15 mm y 15,50 mm considerándose dentro de la sensibilidad media de los aceites esenciales según la escala de Duraffourd.

En la tabla 2 se presentan de la misma manera los datos de las medias de halos de inhibición de las diferentes diluciones del extracto alcohólico de Mentha piperita. A concentración de 5000 ppm este presentó actividad antifúngica nula con una media de $4,5 \mathrm{~mm} \pm 0.57$, mientras que, para las concentraciones de 10000 ppm, 20000 ppm, 30000 ppm, 40000 ppm y 50000 ppm con las medias respectivas de $9 \mathrm{~mm} \pm 0,9,25 \mathrm{~mm} \pm 0,50,10,5 \mathrm{~mm} \pm 0,57,12,25 \mathrm{~mm} \pm 0,5,13 \mathrm{~mm} \pm 0$.

\section{DISCUSIÓN}

La actividad antifúngica se demostró tanto en las diferentes concentraciones del aceite esencial y extracto alcohólico de las hojas de Mentha piperita siendo en concentraciones de 2500 ppm, 4500 ppm, 6500 ppm y 8500 ppm sensibles frente al microorganismo Malassezia furfur ATCC 14521 según la actividad de los aceites esenciales de Duraffourd y para las concentraciones de 10500 ppm y 12500 ppm de sensibilidad media según Duraffourd para el aceite esencial, mientras que para el extracto alcohólico las concentraciones que obtuvieron mayor halo de inhibición y comprueban su actividad antifúngica fueron las de 10000 ppm, 20000 ppm, 30000 ppm, 40000 ppm y 50000 ppm. Por otra parte, la concentración de 5000 ppm fue la única que presentó actividad nula lo que se atribuye a el tipo de muestra utilizada y que el solvente que se usó no extrajo los metabolitos secundarios en la proporción adecuada para demostrar dicha actividad a tal concentración.

A comparación del extracto alcohólico, las diferentes concentraciones de aceite esencial trabajadas dio como resultado halos de inhibición con mayor diámetro esto se debe a que en el aceite esencial son extraídos en mayor proporción los monoterpenos provocantes de la actividad antifúngica los cuales son el mentol y mentona dando como resultados un crecimiento directamente proporcional de los halos de inhibición, es decir a mayor concentración, mayor diámetros de halos de inhibición y mayor efecto antifúngico. 
En general, nuestro trabajo de investigación está en consonancia con las investigaciones realizadas por Tullio Vivian et al. en el año 2019 en su estudio "Evaluación de la actividad antifúngica del aceite esencial de Mentha piperita (Lamiaceae) y su interacción sinérgica con los azoles" en donde se demostró que el aceite esencial ejerce actividad fungicida contra levaduras del género Malassezia, estableciendo que puede servir para el tratamiento en afecciones como la caspa (4).

Además, en el estudio realizado por Rusenova N. et al en el año 2016 sobre la actividad inhibitoria de doce aceites esenciales contra algunos microorganismos mediante el método de difusión por disco concuerdan con el estudio realizado actualmente, probando que el aceite esencial de Mentha piperita posee actividad antifúngica a concentraciones de 2 a 0,008\% (v/v) (6).

Por otra parte, Lee Min Young et al. en el año 2018 realizaron una investigación in vitro con la finalidad de evaluar el efecto de diferentes extractos alcohólicos y aceites esenciales naturales frente al género de Malassezia obteniendo como resultado que los agentes de lavanda y cidra fueron relativamente menor que los de la Mentha piperita en MIC, MFC y método de difusión de disco, llegando así a la conclusión que tantos los extractos alcohólicos como aceite esencial son eficaces para suprimir la actividad de Malassezia furfur causante de la dermatitis seborreica (7).

El estudio demuestra que las mejores concentraciones en el caso del aceite esencial de menta son a partir de 10500 ppm y 12500 ppm y es la mejor opción antes que las concentraciones de extracto alcohólico debido a que en la escala de Duraffourd éstas presentan sensibilidad media, además que en el tipo de muestra y método de extracción utilizado es el indicado para obtener en mayor proporción los monoterpenos responsables de la actividad antifúngica contra Malassezia furfur.

\section{CONCLUSIONES}

Se determinó la actividad antifúngica del aceite esencial de las hojas de M. piperita en diferentes concentraciones, siendo 2500, 4500, 6500 y 8500 ppm medianamente sensible con halos de inhibición de 9 a 14mm, mientras que para 10500 y 12500 ppm la sensibilidad fue mayor con halos de inhibición a partir de $15 \mathrm{~mm}$.

Se evaluó el efecto antifúngico del extracto alcohólico de la hoja Mentha piperita en diferentes concentraciones, siendo 50000 ppm la de mayor sensibilidad con un halo de inhibición de 13 mm mientras que la concentración de 5000 ppm presentó sensibilidad nula.

Se definió estadísticamente que el aceite esencial presentó mayor efecto antifúngico a concentraciones de 10500 a 12500 ppm con halos de inhibición de $15 \mathrm{~mm} \pm$ de 0.58 mientras que el extracto alcohólico presentó mayor sensibilidad en concentración de 50000 ppm con halos de inhibición de $13 \mathrm{~mm} \pm 0.00$ rechazando nuestra hipótesis nula sobre la igualdad de las medias entrelos 
dos grupos (aceite esencial y extracto alcohólico).

\section{REFERENCIAS}

Alexakis, P., \& Siriopoulos, C. (1999). The international stock market crisis of 1997 and the dynamic relationships between asian stock markets: Linear and nonlinear Granger causality tests. Managerial Finance(25), 22-38.

AMA. (2013). American Marketing Association. Obtenido de Journal of Marketing Reseach: https://www.ama.org/publications/JournalOfMarketing/Pages/Current-Issue.aspx

Andreasen, A. (1994). Social Marketing: Its definition and Domain. Journal of Public Policy and Marketing, 13(Spring), 108-114.

Arcotel. (2018). Estadísticas Anuales Líneas Móviles. Recuperado el 26 de Enero de 2018, de Agencia de Regulación y Control de Telecomunicaciones:

http://www.arcotel.gob.ec/servicio-movil-avanzado-sma/

Barboza, A. (2016). Sobre el Método de la interpretación documental y el uso de las imágines en la sociología. Sociedade e Estado, 21(2), 391-414.

Blodgett, J., Lu, L.-C., \& Rose, G. (2001). Ethical Sensitivity to Stakeholder Interest: A CrossCultrural Comparison. Journal of Academy of Marketing Science, 29(2), 190-202.

Chiarelli, J., Marconi, M., Pistani, M., Waingarten, S., \& Knopoff, E. (Junio de 2017). Sistema de farmacovigilancia: conocimiento y actitudes de los médicos del primer nivel de atención y tasa de notificación de efectos adversos para medicación antituberculosis. Revista Americana de Medicina Respiratoria, 17(2).

Claro. (Enero de 2018). Claro. Recuperado el 2 de Febrero de 2018, de Quienes Somos: https://www.claro.com.ec/personas/institucional/quienes-somos/

CNT-EP. (2018). La Corporación Nacional de Telecomunicaciones CNT EP. Recuperado el 2 de Febrero de 2018, de CNT: http://corporativo.cnt.gob.ec/historia-de-las-telecomunicacionesen-el-ecuador/

Consejo General de Colegios Oficiales de Farmacéuticos. (2010). El papel del farmacéutico en la seguridad del paciente. Recuperado el 29 de Marzo de 2021, de El papel del farmacéutico en la seguridad del paciente:

https://www.portalfarma.com/Profesionales/campanaspf/categorias/Documents/Documento s

-Publica/2010_Informe_Tecnico_Seguridad_del_paciente.pdf

Cruz, M., Ruiz, A., Furones, J., \& Palenzuela, I. (2015). Conocimientos sobre farmacovigilancia del personal de estomatología en municipios seleccionados. Revista de Ciencias Médicas La Habana, 21(3).

Drucker, P. (1984). The new meaning of corporate social responsi-bility. California Management Review, XXVI(2), 53-63.

Gómez, P. (2003). La gestión de marketing de ciudades y áreas metropolitanas: de la orientación al producto a la orientación al marketing. Cuadernos de Gestión, 3(1), 11-25.

Gordhon, Y., \& Pedayachee, N. (2020). Evaluating the knowledge, attitudes and practices of 
healthcare workers towards adverse drug reaction reporting at a public tertiary hospital in Johannesburg. International Journal of Africa Nursing Sciences, 12.

Green Paper. (2011). Green Paper on corporate social responsibility. Recuperado el 2018 de Enero de 2018, de Europa: http://europa.eu/legislation

Hunt, S. (1983). General theories and the fundamentalexplananda of marketing. Journal of Marketing, 47(4), 9-17.

Jiménez, G., Debesa, F., González, B., Ávila, J., \& Pérez, J. (2006). El Sistema Cubano de Farmacovigilancia, seis años de experiencia en la detección de efectos adversos. Rev Cubana Farm, 40(1), 1-8. Obtenido de http://scielo.sld.cu/pdf/far/v40n1/far02106.pdf

Jiménez, G., García, A., Gálvez, A., Alfonso, I., Lara, M., \& Calvo, D. (2014). Medicamentos notificados como productores de reacciones adversas graves en Cuba en un período de diez años. Revista Cubana de Salud Pública, 40(4), 263-275. Obtenido de https://www.medigraphic.com/pdfs/revcubsalpub/csp-2014/csp144c.pdf

Kotler, P., \& Levy, S. (1969). Broading the concept of Marketing. Journal of Marketing, 33(January), 10-15.

Kotler, P., \& Zaltman, G. (1971). Social marketing: An approach toplanned social change. Journal of Marketing, 35, 3-12.

Lideres. (Enero de 2014). Revista Lideres. Recuperado el 12 de Enero de 2018, de En 20 años, la telefonía móvil superó las expectativas: http://www.revistalideres.ec/lideres/20-anostelefonia-movil-supero.html.

Maignan, I., \& Ferrell, O. (2004). Corporate social responsibility and marketing: an integrative framework. Journal of the Academy of Marketing Science, 32(1), 19-23.

Mata, J., Ortiz, M., Islas, H., Diaz, M., De León, V., \& Tenorio, L. (Octubre-diciembre de 2017). Impacto de una intervención educativa en los conocimientos en farmacovigilancia y en el reporte de reacciones adversas a los medicamentos de profesionales de la salud en un hospital público de segundo nivel de atención en el Estado de México, México. Revista Mexicana de Ciencias Farmacéuticas, 48(4).

Maza, J., Aguilar, L., \& Mendoza, J. (2018). Farmacovigilancia: un paso importante en la seguridad del paciente. Medigraphic, 72(1), 47-53.

Miron, D., Petcu, M., \& Sobolevschi, M. (2011). Corporate Social Responsibility and sustainable competitiveness. Amfiteatru Economic Journal, A.S.E.(29), 163-180.

Muberra, G., \& Perihan, E. (Enero de 2019). Healthcare professionals pharmacovigilance knowledge and adverse drug reaction reporting behavior and factors determining the reporting rates. Journal of Drug Assessment, 8(1).

Ramos, J., \& Periáñez, I. (2003). Delimitación del Marketing con Causa o Marketing Social Corporativo mediante el análisis de empresas que realizan acciones de responsabilidad social. Cuadernos de Gestión, 3(1), 65-82.

Romero, K. (Abril-Junio de 2018). El conocimiento de la Farmacología en el profesional de enfermería. Enfermería Investiga, Investigación, Vinculación, Docencia y Gestión, 3(2). 
Ruiz, A., García, A., Jiménez, G., Alfonso, I., Pérez, B., \& Carrazana, A. (2013). Farmacovigilancia de fitofármacos y apifármacos en Cuba durante 2006-2010. Revista Cubana de Plantas Medicinales, 18(2), 173-186. Obtenido de http://scielo.sld.cu/pdf/pla/v18n2/pla02213.pdf

Sáez, M., Sánchez, N., Jiménez, S., Alonso, N., \& Valverde, J. (Marzo-Abril de 2016). Tratamiento del dolor en el anciano: opioides y adyuvantes. Revista de la Sociedad Española del Dolor, 23(2).

Telefónica S.A. (2018). Movistar. Recuperado el 3 de Febrero de 2018, de Historia de Movistar: https://www.movistar.com.ec/

Torres, B. (2006). Metodología de la Investigación. Mexico D.F: Pearson.

Trabanca, Y., Jiménez, G., Alfonso, i., Pavón, K., \& Albear, F. (Marzo-Abril de 2018). Implementación del Programa de Notificación de Efectos Adversos por Pacientes en Guantánamo. Revista de Información Científica, 97(2).

Vaaland, T., Heide, M., \& Grønhaug, K. (2008). Corporate social responsibility: investigating theory and research in the marketing context. European Journal of Marketing, (9/10), 927953.

Vázquez, M. (2011). Investigación en educación matemática: objetivos, cambios, criterios, método y difusión. Education Siglo XXI, 29(2), 173-198.

Vicente, M. Azucena; Mediano, Lucía; . (2002). Propuestas para una segmentación estragégica del mercado ecológico . Cuadernos de Gestión, 2(1).WBCSD. (2012). World Business Council for Sustainable Development,. Recuperado el 15 de Enero de 2018, de . Corporate social responsibility: Meeting changing expectations [pdf].: http://www.wbcsd.org 